\title{
c-di-AMP-Regulated $\mathrm{K}^{+}$Importer KtrAB Affects Biofilm Formation, Stress Response, and SpeB Expression in Streptococcus pyogenes
}

\author{
Sabrina Faozia, ${ }^{\text {T Tazin Fahmi, }}$ Gary C. Port, ${ }^{\text {* }}$ (D) Kyu Hong Cho ${ }^{a}$ \\ aDepartment of Biology, Indiana State University, Terre Haute, Indiana, USA
}

\begin{abstract}
The second messenger cyclic di-AMP (c-di-AMP) controls biofilm formation, stress response, and virulence in Streptococcus pyogenes. The deletion of the cdi-AMP synthase gene, dacA, results in pleiotropic effects including reduced expression of the secreted protease SpeB. Here, we report a role for $\mathrm{K}^{+}$transport in $\mathrm{c}$-diAMP-mediated SpeB expression. The deletion of $k t r B$ in the $\triangle$ dacA mutant restores SpeB expression. KtrB is a subunit of the $\mathrm{K}^{+}$transport system KtrAB that forms a putative high-affinity $\mathrm{K}^{+}$importer. KtrB forms a membrane $\mathrm{K}^{+}$channel, and KtrA acts as a cytosolic gating protein that controls the transport capacity of the system by binding ligands including c-di-AMP. SpeB induction in the $\triangle d a c A$ mutant by $\mathrm{K}^{+}$specific ionophore treatment also supports the importance of cellular $\mathrm{K}^{+}$balance in SpeB production. The $\Delta d a c A \Delta k t r B$ double deletion mutant not only produces wildtype levels of SpeB but also partially or fully reverts the defective $\Delta$ dacA phenotypes of biofilm formation and stress responses, suggesting that many $\triangle d a c A$ phenotypes are due to cellular $\mathrm{K}^{+}$imbalance. However, the null pathogenicity of the $\triangle$ dacA mutant in a murine subcutaneous infection model is not restored by ktrB deletion, suggesting that c-di-AMP controls not only cellular $\mathrm{K}^{+}$balance but also other metabolic and/or virulence pathways. The deletion of other putative $\mathrm{K}^{+}$importer genes, kup and $\operatorname{kim} A$, does not phenocopy the deletion of $k \operatorname{tr} B$ regarding SpeB induction in the $\triangle$ dacA mutant, suggesting that $\mathrm{KtrAB}$ is the primary $\mathrm{K}^{+}$importer that is responsible for controlling cellular $\mathrm{K}^{+}$levels under laboratory growth conditions.
\end{abstract}

KEYWORDS Streptococcus pyogenes, c-di-AMP, DacA, SpeB, KtrAB, Kup, KimA

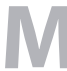
ost organisms produce cyclic nucleotide second messengers that regulate cellular activities by binding to effector molecules such as proteins or RNAs. Cyclic nucleotide-involved signaling pathways sense environmental changes, such as temperature, nutrition, $\mathrm{pH}$, and other stressors, and transmit the signals to effector molecules (1-4). Many bacteria have been shown to produce several different cyclic dinucleotides, such as c-di-AMP (cyclic di-AMP), c-di-GMP (cyclic di-GMP), and cGAMP (cyclic GMP-AMP). c-di-AMP, produced by many bacteria and archaea (5), is involved in controlling diverse cellular processes, such as fatty acid biosynthesis (6), DNA integrity detection (7-9), and cell wall homeostasis (10-13), in a bacterium-specific manner. However, more than a decade of research has shown that a major role of c-di-AMP in most bacteria is in maintaining proper turgor pressure by controlling the activity of ion and/or osmolyte transporters (14-16).

Many important pathogens, including Streptococcus species (S. pyogenes, S. agalactiae, and S. mutans), Staphylococcus aureus, Mycobacterium tuberculosis, and Listeria monocytogenes, appear to export c-di-AMP into the environment. When these pathogens are internalized into their host cells, the secreted c-di-AMP is detected by the host cell's STING (STimulator of INterferon Gene) $(5,17)$. STING also senses 2' 3 '-cGAMP
Citation Faozia S, Fahmi T, Port GC, Cho KH 2021. C-di-AMP-regulated $\mathrm{K}^{+}$importer KtrAB affects biofilm formation, stress response, and SpeB expression in Streptococcus pyogenes. Infect Immun 89:e00317-20. https://doi.org/10 .1128/IAl.00317-20.

Editor Nancy E. Freitag, University of Illinois at Chicago

Copyright $\odot 2021$ American Society for Microbiology. All Rights Reserved. Address correspondence to Kyu Hong Cho, kyuhong.cho@indstate.edu.

* Present address: Gary C. Port, Elanco Animal Health, Natural Products Fermentation, Indianapolis, Indiana, USA

Received 29 May 2020

Returned for modification 26 July 2020 Accepted 14 January 2021

Accepted manuscript posted online 19 January 2021

Published 17 March 202 
( $2^{\prime} 3^{\prime}$ cyclic GMP AMP), another cyclic dinucleotide produced by the host enzyme cGAS (cyclic GMP AMP synthase) that is activated by bacterial DNA in the host cytosol $(5,17)$. When sensing these c-dinucleotides, STING activates type I interferon production. Thus, c-di-AMP regulates not only bacterial but also host cellular processes during infection. However, the detailed role of c-di-AMP during infection is largely unknown.

S. pyogenes, also known as group A Streptococcus (GAS), is a Gram-positive pathogen that generally causes noninvasive diseases, such as strep throat and impetigo. However, these superficial infections sometimes develop serious diseases, such as rheumatic heart disease, streptococcal toxic shock syndrome, poststreptococcal glomerulonephritis, and necrotizing fasciitis. A minimum global burden by $S$. pyogenes infection is estimated at over 18 million cases of severe diseases, resulting in over half a million annual deaths (18). Despite the dire consequences of this pathogen, commercial vaccines are not yet available.

Our recent study has revealed that c-di-AMP regulates diverse cellular traits and virulence of $S$. pyogenes (18). When dacA, the only c-di-AMP synthase gene in the S. pyogenes chromosome, is deleted, the $\triangle$ dacA mutant strain displays many defective phenotypes, including increased lag time for growth, reduced biofilm formation and SpeB expression, and increased sensitivity to stressors such as high salt concentrations, low $\mathrm{pH}$, reactive oxygen, and the cell wall-targeting antibiotic ampicillin. Here, we show that the null mutation of a high-affinity $\mathrm{K}^{+}$channel protein $\mathrm{KtrB}$ in the $\triangle$ dacA mutant reverts most of these defective phenotypes, suggesting that the majority of the $\triangle$ dacA phenotypes result from KtrAB malfunction caused by the absence of c-di-AMP. However, the deletion of $k \operatorname{tr} B$ in the $\triangle d a c A$ mutant was unable to restore virulence in a murine model, suggesting that c-di-AMP also plays roles in cell physiology and/or virulence other than $\mathrm{K}^{+}$transport in S. pyogenes.

\section{RESULTS}

Mutation of the $\mathrm{K}^{+}$importer subunit KtrB restores the SpeB expression of $\triangle$ dacA mutant. We have previously reported that the deletion of the c-di-AMP synthase gene, $d a c A$, abolishes the ability of $S$. pyogenes to produce the secreted cysteine protease SpeB (18). Unexpectedly, however, one of the $18 \Delta$ dacA strains created by the dacA in-frame deletion process showed $\mathrm{SpeB}^{+}$. Whole-genome sequencing of this mutant revealed a single base pair deletion in ktrB (Spy_0327 based on the SF370 reference; L897_01540 in HSC5). KtrB is a subunit of the KtrAB system, known to be a highaffinity $\mathrm{K}^{+}$importer (19-21). KtrB is a membrane-integrated protein that forms a $\mathrm{K}^{+}$ channel and interacts with the cytosolic protein KtrA (Spy_0326; L897_01535 in HSC5) that controls the transport activity of KtrB (22). KtrA orthologs, KtrA in S. aureus, CabP in S. pneumoniae, and CapPA in S. mutans, are known c-di-AMP-binding proteins (23-25). In S. pyogenes, ktrA and ktrB form a bicistronic operon in the order of ktrBA (Fig. 1), and their coexpression was confirmed by total RNA sequencing (26). The $\Delta$ dacA $\Delta k \operatorname{tr} B^{\text {sup }}$ suppressor mutant contained a single-nucleotide deletion within a homopolymeric stretch of eight thymines in ktrB, resulting in a frameshift mutation (Fig. 1).

To confirm that ktrB is responsible for the suppression of the SpeB phenotype observed in the $\Delta d a c A \Delta k t r B^{\text {sup }}$ strain, an in-frame deletion of $k t r B$ was generated in the $\Delta$ dacA mutant, and the SpeB activity of the $\Delta$ dac $A \Delta k \operatorname{tr} B$ mutant was examined. As expected, the $\Delta$ dacA $\Delta k \operatorname{tr} B$ mutant produced $\mathrm{SpeB}$, similar to the $\Delta$ dacA $\Delta k \operatorname{tr} B^{\text {sup }}$ mutant and the wild type (Fig. 2). To further confirm the role of the KtrAB system in regulating c-di-AMP-mediated SpeB regulation, the ktrA gene was subsequently deleted in the $\Delta$ dacA mutant, and the resulting $\Delta d a c A \Delta k \operatorname{tr} A$ mutant also produced SpeB, although to a lesser extent than the wild type (Fig. $2 C$ ). SpeB activity was examined in $\Delta k t r A$ and $\Delta k t r B$ single-gene deletion mutants, and their SpeB activity was similar to that of the wild type (Fig. 2D). Quantitative reverse transcription-PCR (qRT-PCR) analysis measuring speB transcript levels in the cells demonstrated that the SpeB activity change in the $\triangle$ dacA $\Delta k t r B$ and $\Delta$ dacA $\Delta k \operatorname{tr} A$ mutants occurs at the transcriptional level (Fig. 3). 


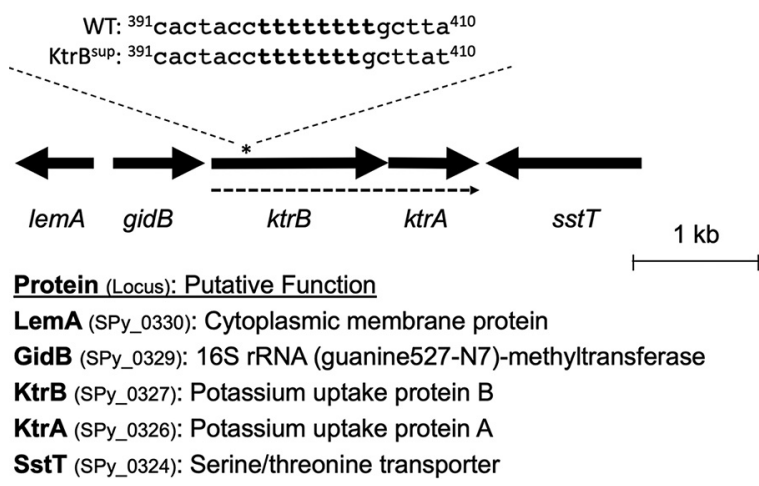

FIG 1 ktrB genomic context and the mutation site in $k \operatorname{tr} B$ of the $\Delta d a c A \Delta k \operatorname{tr} B^{\text {sup }}$ suppressor mutant. Each arrow indicates an individual open reading frame and its orientation. $k \operatorname{tr} B$ is coexpressed with the downstream gene $k t r A$, which is shown with a dotted arrow under the ktrB and ktrA genes. The predicted proteins encoded by these open reading frames and their putative functions are shown below the gene organization. The asterisk shows the location of the mutation site in $k \operatorname{tr} B$ in the $\Delta d a c A \Delta k \operatorname{tr} B^{\text {sup }}$ suppressor mutant. In the mutant, one thymidine out of eight consecutive thymidines is deleted.

Mutation of ktrB reverts the defects of biofilm formation and stress response of $\Delta$ dacA mutant to levels equal to or near that of the wild type. The deletion of dacA results in various defective growth characteristics, such as increased lag phase, decreased biofilm formation, and increased sensitivity to low $\mathrm{pH}$, high-salt concentrations, oxidative stress, and cell wall-targeting antibiotics (18). When ktrB was mutated in the $\triangle$ dacA mutant, these defective phenotypes were partially or fully reverted to those of the wild type (Fig. 4). The lag time of the $\Delta d a c A$ mutant is almost twice that of the wild type ( $4.5 \mathrm{~h}$ versus $2.5 \mathrm{~h}$ in Todd-Hewitt medium supplemented with $0.2 \%$ yeast extract [THY medium]), and the $\triangle$ dacA $\Delta k \operatorname{tr} B$ mutant decreased its lag time to that of the wild type $(2.5 \mathrm{~h})$. The $\Delta$ dacA mutant cannot form a biofilm, but the $\triangle$ dacA $\Delta k \operatorname{tr} B$ mutant partially regained the ability to form a biofilm (50\% as much biofilm as the wild type) (Fig. 4A). The $\triangle$ dacA mutant cannot grow in THY or $\mathrm{C}$ medium whose initial $\mathrm{pH}$ is adjusted to 6.0. In contrast, the $\triangle$ dacA $\Delta k \operatorname{tr} B$ mutant reached an optical density at 600 $\mathrm{nm}\left(\mathrm{OD}_{600}\right)$ of approximately $66 \%$ of wild-type levels in acidified THY medium but still did not grow in acidified C medium (Fig. 4B). The $\Delta$ dacA mutant cannot grow or grows very poorly in THY medium containing additional $0.1 \mathrm{M} \mathrm{KCl}$ or $\mathrm{NaCl}$. However, the $\Delta$ dacA $\Delta k \operatorname{trB}$ mutant grew to nearly wild-type levels under both conditions (Fig. $4 C$ ). The $\triangle$ dacA mutant shows higher sensitivity to reactive oxygen species than the wild type under both short-term and long-term exposure conditions. In contrast, the $\Delta d a c A$ $\Delta k t r B$ mutant showed decreased sensitivity to reactive oxygen species compared to the $\Delta d a c A$ mutant (Fig. 4D). The $\triangle d a c A$ mutant is more resistant to $\mathrm{PlyC}$, as seen by a decrease in cell lysis compared to that of the wild type, whereas the $\Delta \operatorname{dac} A \Delta k \operatorname{tr} B$ mu-

A)

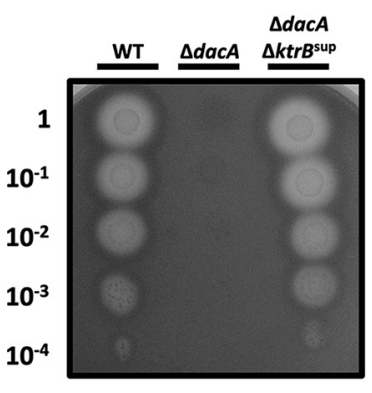

B)

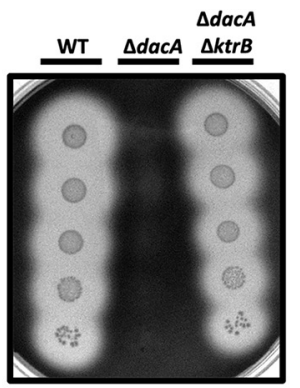

C)

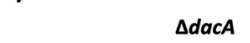

D)

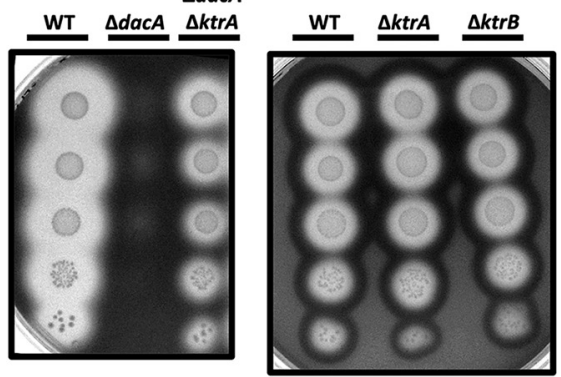

FIG 2 Deletion of $k \operatorname{tr} B$ restores the speB activity of $\Delta$ dacA mutant. The activity of the secreted protease SpeB is shown on protease indicator plates. Strains were grown overnight and spotted ( $2 \mu \mathrm{l}$ ) onto protease indicator agar plates after serial dilution. Protease activity displays a clear zone around the spotted cells after incubation. Strain names are shown above the images, and dilution degrees of the spotted cultures are indicated at the left side of the images. Plates were incubated for $24 \mathrm{~h}$. 


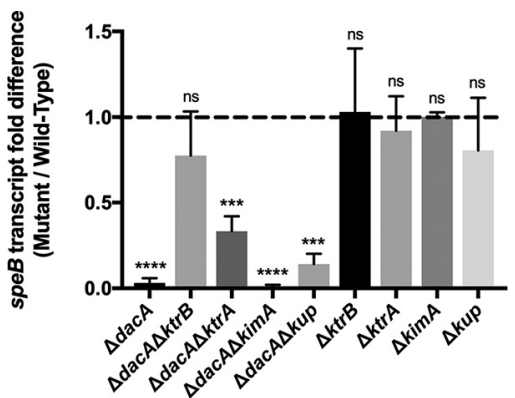

FIG 3 SpeB activity variations of the mutants were caused at the transcriptional level. The relative abundance of the $s p e B$ transcript during the stationary-phase growth in mutants was determined using qRT-PCR and compared to that of the wild type. Each column represents the speB transcript abundance in a mutant relative to that in the wild type. Shown are the means and standard deviations from three independent experiments. Asterisks indicate the significance of the difference between a mutant and the wild type, as calculated by one-way analysis of variance (ANOVA) followed by Dunnett's multiplecomparison test (ns, not significant; ${ }^{*}, P<0.05 ;{ }^{* *}, P<0.01$; ${ }^{* * *}, P<0.001$; and ${ }^{* * * *}, P<0.0001$ ).

tant displayed wild-type levels of resistance to PlyC (Fig. 4E). The sensitivity of the $\triangle$ dacA mutant to sublethal levels of ampicillin treatment is much higher than that of the wild type, whereas the sensitivity of the $\Delta$ dac $A \Delta k \operatorname{tr} B$ mutant was comparable to that of the wild type (Fig. 4F).

Mutation of $k t r B$ does not restore the attenuated virulence of the $\triangle$ dacA mutant. The $\triangle$ dacA mutant displays no virulence in a murine subcutaneous infection model, as seen by the absence of a lesion (18). The virulence of the $\Delta d a c A \Delta k t r B$ mutant was examined using the same soft-tissue infection model, and, surprisingly, none of the mice injected with the $\Delta d a c A \Delta k \operatorname{tr} B$ mutant developed lesions (Fig. 5). Thus,

A)

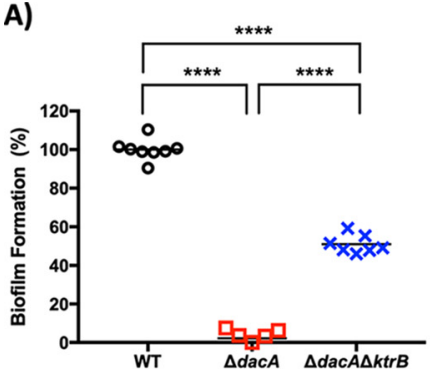

D)

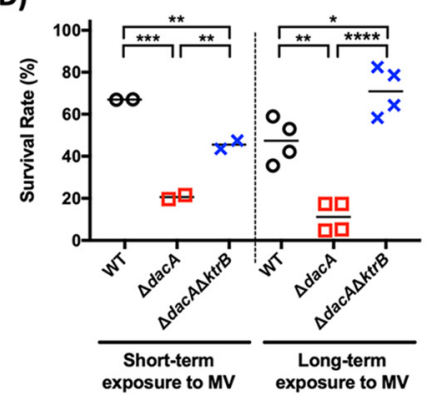

B)

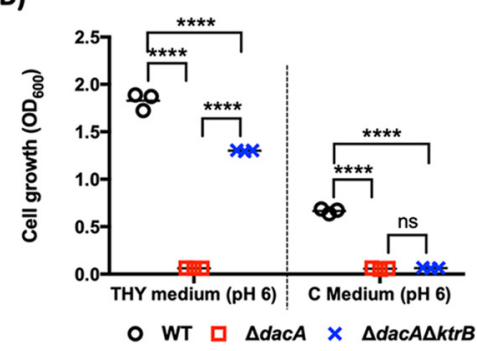

E)

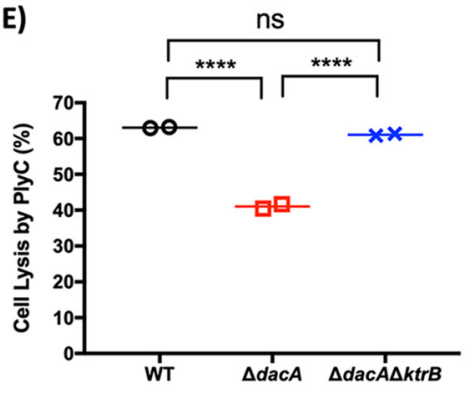

C)

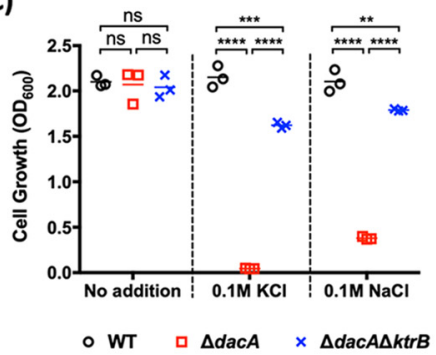

F)

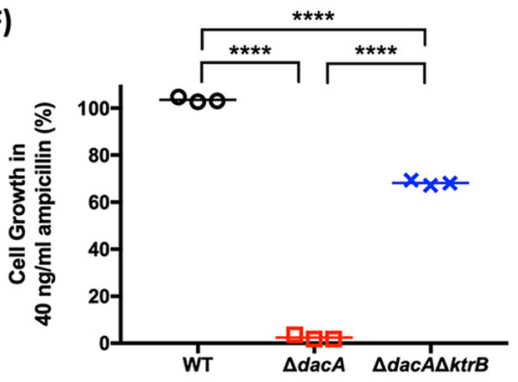

FIG 4 KtrB mutation either fully or partially reverts defective growth phenotypes caused by dacA deletion. (A) Biofilm formation ability of $S$. pyogenes strains. (B) The growth of $S$. pyogenes $\left(\mathrm{OD}_{600}\right)$ at $8 \mathrm{~h}$ postinoculation in acidified THY or $\mathrm{C}$ medium ( $\mathrm{pH} 6.0$ ). (C) The growth of $S$. pyogenes $\left(\mathrm{OD}_{600}\right)$ at $8 \mathrm{~h}$ postinoculation in THY medium added with $0.1 \mathrm{M} \mathrm{KCl}$ or $\mathrm{NaCl}$. (D) Relative viability of $S$. pyogenes strains after being exposed to oxidative stress caused by methyl viologen (MV; $50 \mathrm{mM}$ for $3 \mathrm{~h}$ in short-term exposure, $1.7 \mathrm{mM}$ for $18 \mathrm{~h}$ in long-term exposure). (E) Cell lysis by the cell wall-hydrolyzing enzyme PlyC. Each strain was incubated with PlyC $(3,000 \mathrm{U} / \mathrm{ml})$ at $37^{\circ} \mathrm{C}$. The turbidity $\left(\mathrm{OD}_{600}\right)$ of each strain at $6 \mathrm{~h}$ postincubation was measured to determine the degree of cell lysis. $(\mathrm{F})$ Relative cell growth $\left(\mathrm{OD}_{600}\right)$ by comparing the growths with or without sublethal levels of ampicillin ( $\left.40 \mathrm{ng} / \mathrm{ml}\right)$. The horizontal bar in each data set is the mean value of the data points. The significance of the difference between values from two different strains was calculated by one-way ANOVA with Tukey's multiple-comparison test (ns, not significant; ${ }^{*}, P<0.05 ;{ }^{* *}, P<0.01 ;{ }^{* *}, P<0.001 ;$ and ****, $P<0.0001)$. 


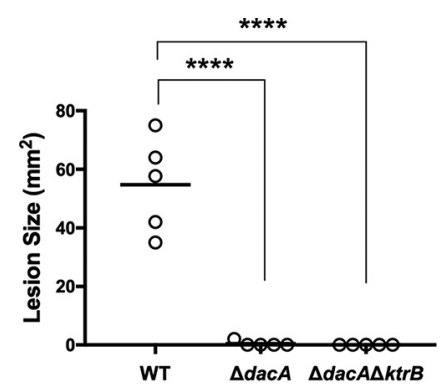

FIG 5 Deletion of $k \operatorname{tr} B$ does not revert the severely attenuated virulence of the $\Delta$ dac $A$ mutant. The ability of wild-type HSC5 (A), $\Delta \operatorname{dacA}(\mathrm{B})$, and $\Delta \operatorname{dac} A \Delta k \operatorname{tr} B(\mathrm{C})$ strains to cause lesions in a murine subcutaneous infection model is shown. The virulence of each strain was evaluated by measuring the area of the lesion formed on day 3 postinfection when lesion sizes were maximal. Each circle represents the size of a lesion formed by an injection of the wild-type, $\Delta$ dacA, or $\Delta$ dacA $\Delta k \operatorname{tr} B$ strain. Each solid bar indicates the mean value of ulcer sizes. The asterisk marks above each bracket indicate the significance of difference $\left.{ }^{* * * *}, P<0.0001\right)$ between each mutant and the wild type as calculated by the Mann-Whitney $U$ test statistic.

even though many phenotypes of the $\triangle$ dacA mutant were reverted to those of the wild type by ktrB mutation (Fig. 4), the virulence loss of the $\triangle$ dacA mutant was not reverted by the same mutation.

Valinomycin and gramicidin ionophore treatment mimics the effect of ktrB mutation on the restoration of SpeB activity of the $\Delta$ dacA mutant. lonophores are small hydrophobic lipid-soluble molecules that transport ions across cell membranes. Because ionophores are not coupled to energy sources, they only transport ions down their electrochemical gradient and are used as antibiotics due to their ability to collapse ion gradients across cellular membranes. Since the deletion of $k \operatorname{tr} B$ restores SpeB production in the $\triangle d a c A$ mutant, we examined the effect of the ionophores that disturb cellular $\mathrm{K}^{+}$balance, such as valinomycin and gramicidin, on SpeB production (27). Valinomycin is a carrier ionophore that transports $\mathrm{K}^{+}$across the membrane. Gramicidin is a channel-forming ionophore that transports small inorganic monovalent cations, such as $\mathrm{K}^{+}$and $\mathrm{Na}^{+}$, across the membrane (27). Since both of these ionophores have growth inhibition effects, sub-growth-inhibitory concentrations of these ionophores were determined by monitoring S. pyogenes growth $\left(\mathrm{OD}_{600}\right)$ at different concentrations. A concentration of $1 \mu \mathrm{g} / \mathrm{ml}$ valinomycin or $0.5 \mu \mathrm{g} / \mathrm{ml}$ gramicidin has little to no effect on the growth of wild-type HSC5 in liquid culture. When tested on solid media, these conditions also minimally affected the growth and SpeB activity of the wild type (Fig. 6). When the $\triangle$ dacA mutant was treated with the ionophores, it regained the ability to produce SpeB (Fig. 6), mimicking the effect of ktrB mutation on SpeB activity of the $\Delta$ dacA mutant (Fig. 2). When the $\Delta$ dacA $\Delta k t r B$ mutant was treated with ionophores, it did not significantly change SpeB activity (Fig. 6).

GdpP and Pde2 are two c-di-AMP phosphodiesterases identified in S. pyogenes (18). Similar to the $\triangle$ dacA mutant, the $\Delta p d e 2$ mutant does not produce SpeB (18). However, unlike the $\Delta d a c A$ mutant, the $\Delta p d e 2$ mutant did not show SpeB activity when treated with the ionophores (Fig. 6). This result indicates that the underlying mechanism causing the SpeB null phenotype of the $\Delta p d e 2$ mutant is different from that of the $\Delta$ dacA mutant.

Deletion of putative $\mathrm{K}^{+}$importer gene kup or kimA does not phenocopy ktrB deletion. The $S$. pyogenes genome encodes homologs of at least two additional $\mathrm{K}^{+}$ importers other than KtrAB, which include a Kup homolog (SPy_1414 in SF370, L897_05730 in HSC5) and a KimA homolog (SPy_2088 in SF370, L897_08830 in HSC5). In-frame deletion mutants of each of these $\mathrm{K}^{+}$transporter homologs were generated in the $\Delta$ dacA mutant, and SpeB activity of the resulting $\Delta$ dacA $\Delta$ kup and $\Delta$ dacA $\Delta$ kim $A$ mutants was examined. The $\Delta d a c A \Delta k u p$ mutant showed very low SpeB activity on a protease agar plate following extended incubation (Fig. 7). Similarly, the $\triangle \operatorname{dac} A \Delta$ kimA mutant did not exhibit SpeB activity. These results indicate that the role of KimA and Kup in the $\triangle d a c A$ mutant under the in vitro growth conditions was not as crucial as that of KtrAB. SpeB activity of $\Delta k i m A$ and $\Delta k u p$ single-gene deletion mutants was 


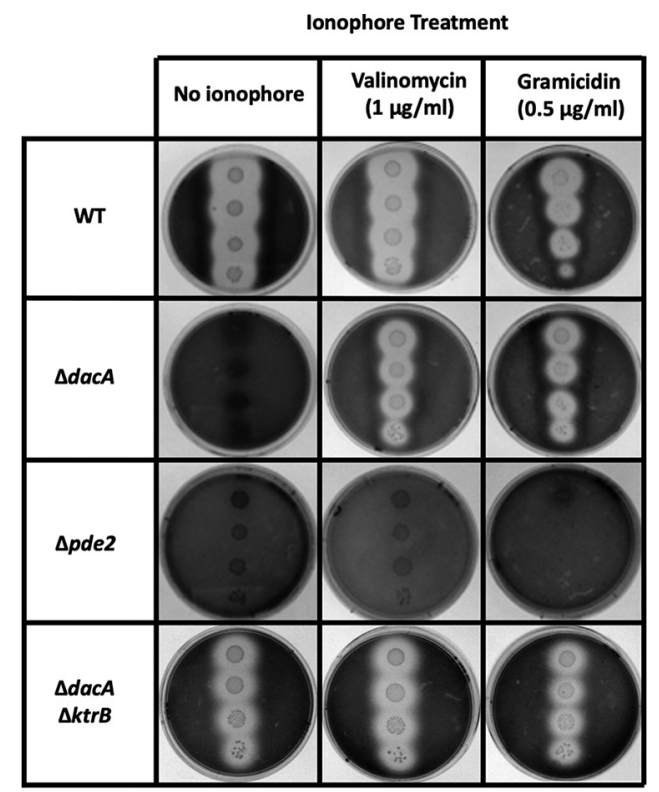

FIG 6 lonophores specific to $\mathrm{K}^{+}$(valinomycin) and monovalent cations (gramicidin) restore SpeB activity of the $\triangle$ dacA mutant to the wild-type level. The effect of ionophores on SpeB activity of $S$. pyogenes strains was observed. Overnight cultures of $S$. pyogenes strains were serially diluted with THY medium and spotted onto protease indicator plates containing no ionophore, valinomycin $(1 \mu \mathrm{g} /$ $\mathrm{ml})$, or gramicidin $(0.5 \mu \mathrm{g} / \mathrm{ml})$. Culture dilution degrees were $10^{1}, 10^{2}, 10^{3}$, and $10^{4}$ from the top.

comparable to that of the wild type (Fig. 7). qRT-PCR analysis measuring the $s p e B$ transcript levels in the cells demonstrate that the SpeB activity change in these mutants occurred at the transcriptional level (Fig. 3).

\section{DISCUSSION}

The potassium ion $\left(\mathrm{K}^{+}\right)$is the most abundant intracellular cation and has a primary role in turgor compensation and osmotic adjustment $(28,29)$. Thus, controlling cellular $\mathrm{K}^{+}$levels through $\mathrm{K}^{+}$transporters is crucial for the survival of cells in diverse osmotic environments. It has been shown in Gram-positive bacteria that c-di-AMP inhibits transporters that maintain turgor pressure and osmotic adjustment, such as potassium transporters and/or glycine-betaine transporters (14-16, 30, 31). For example, c-di-AMP inhibits the activity of the multiple potassium transporters, the KtrAB system, the KtrCD system, and KimA in B. subtilis and the KupA and KupB potassium transporters in Lactococcus lactis $(30,31)$.

In this study, KtrB, the channel component of the $\mathrm{K}^{+}$importer KrtAB system, was identified as a suppressor of the SpeB null phenotype of the $\triangle d a c A$ mutant, suggesting

A)

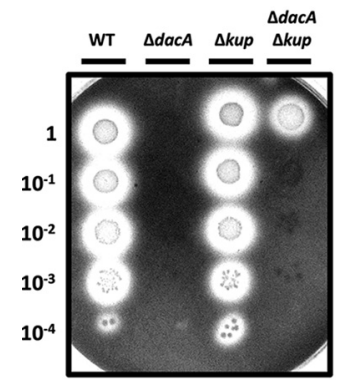

B)

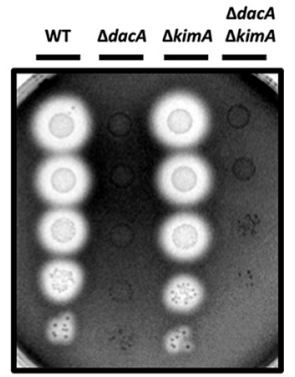

FIG 7 Deletion of a putative $\mathrm{K}^{+}$importer gene, kup or kimA, does not phenocopy ktrB deletion in the $\triangle$ dacA mutant. Unlike the deletion of $k t r B$, the deletion of another $\mathrm{K}^{+}$importer gene, kup or $\operatorname{kimA}$, did not restore the SpeB activity of the $\triangle d a c A$ mutant to the wild-type level. Strain names are shown above the pictures, and the dilution degrees of the cultures are indicated at the left side of the images. The protease indicator plates were incubated for $48 \mathrm{~h}$ in this experiment (most strains developed zones of clearance in $24 \mathrm{~h}$, but the $\Delta$ dacA $\Delta$ kup strain took $48 \mathrm{~h}$ to become visible). 


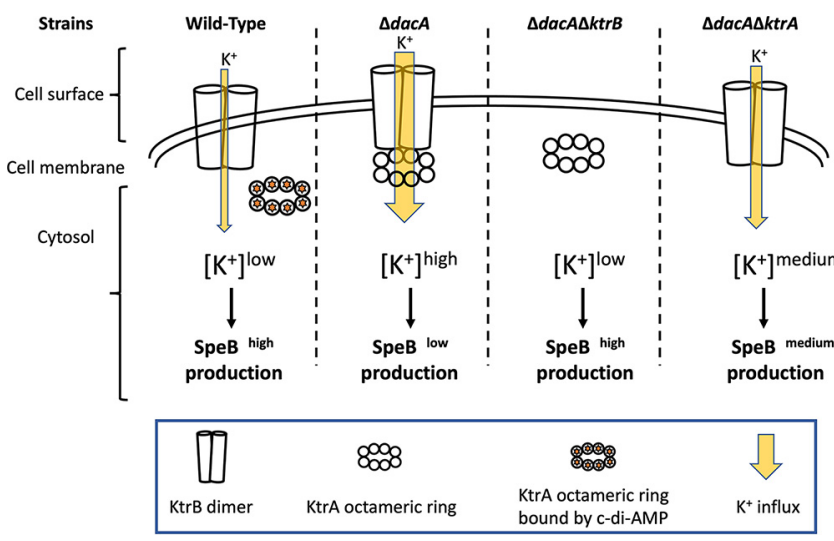

FIG 8 Model explaining the relationship between the activity of the KtrAB system and SpeB production in S. pyogenes. Interactions between c-di-AMP and the KtrAB system and their effect on SpeB expression in the wild type (WT), $\Delta d a c A, \Delta d a c A \Delta k t r B$, and $\Delta d a c A \Delta k \operatorname{trA}$ strains are shown.

that the control of cellular $\mathrm{K}^{+}$concentration connects c-di-AMP signal transduction and SpeB production. KtrAB is a member of the Trk/Ktr/HKT $\mathrm{K}^{+}$transporter superfamily that consists of uniporters and symporters $\left(\mathrm{K}^{+} / \mathrm{Na}^{+}\right.$or $\left.\mathrm{K}^{+} / \mathrm{H}^{+}\right)$(32). KtrAB appears to be a high-affinity $\mathrm{K}^{+}$importer, as the $\mathrm{Km}$ value of the $\mathrm{Ktr} A B$ system in $B$. subtilis for $\mathrm{K}^{+}$is in the micromolar range (19-21). The KtrAB system is composed of a homodimeric KtrB membrane protein complex and a cytosolic KtrA octameric ring (22). Each KtrB subunit forms a potassium channel, so deletion of $k t r B$ abolishes the $\mathrm{K}^{+}$transport ability of the KtrAB system. KtrA is a gating component regulating KtrB activity (22). KtrA contains regulator of conductance of potassium (RCK) domains at the amino terminus (RCK_N) and carboxy terminus (RCK_C). ATP and ADP bind to the RCK_N domain. When ATP binds to the domain, the $\mathrm{K}^{+}$transport ability of KtrAB increases, but when ADP binds, the ability decreases. It has been shown that KtrA orthologs in S. aureus (KtrA), S. pneumoniae (CabP), and S. mutans (CapPA) are c-di-AMP-binding proteins (23-25). c-di-AMP binds to the RCK_C domain of KtrA and inhibits the $\mathrm{K}^{+}$transport ability of the KtrAB system (23). KtrA bound by c-di-AMP does not interact with KtrB in S. pneumoniae (25). $\mathrm{KtrB}$ without $\mathrm{Ktr} A$ has almost half of its full $\mathrm{K}^{+}$transport activity in B. subtilis (32).

A model for the interaction of c-di-AMP and $\mathrm{K}^{+}$and SpeB expression is shown in Fig. 8. As the deletion of dacA abolishes the ability of $S$. pyogenes to synthesize c-di-AMP (18), the KtrA octameric ring in the absence of c-di-AMP likely interacts with the KrtB dimer, thereby promoting $\mathrm{K}^{+}$import (Fig. 8, $\triangle$ dacA mutant) (25). Alternative $\mathrm{K}^{+}$transporters and glycinebetaine transporters may import additional $\mathrm{K}^{+}$and glycine-betaine in the absence of $\mathrm{c}$-diAMP (14-16). This increased cellular $\mathrm{K}^{+}$concentration may trigger repression of SpeB expression via a decrease in speB transcription through a currently undefined mechanism. Removal of the $\mathrm{K}^{+}$channel $\mathrm{KtrB}$ in a c-di-AMP-devoid strain likely leads to a decrease in intracellular $\mathrm{K}^{+}$and subsequent restoration of SpeB expression via an increase in speB transcription (Fig. 8, $\Delta$ dacA $\Delta$ ktrB mutant). This model is further supported through exposure to ionophores, as a sublethal treatment of the $\triangle d a c A$ mutant with valinomycin or gramicidin presumably leads to an efflux of $\mathrm{K}^{+}$from the cells and restoration of SpeB production in the $\Delta$ dacA mutant (Fig. 6). However, ionophore treatment of the $\Delta$ dacA $\Delta k t r B$ mutant has no effect on SpeB production, because the $\triangle$ dac $A$ $\Delta k t r B$ mutant presumably already has low cellular $\mathrm{K}^{+}$(Fig. 6). The $\Delta$ dacA $\Delta k t r A$ mutant appears to import more $\mathrm{K}^{+}$ than the wild type, since SpeB activity of the strain is less than that of the wild type (Fig. $8, \Delta$ dacA $\Delta k$ trA mutant). Lower $\mathrm{K}^{+}$import than that of the wild type does not appear to influence SpeB expression, because all of the single $\mathrm{K}^{+}$transporter gene deletion mutants, $\Delta k \operatorname{tr} A, \Delta k t r B, \Delta k u p$, and $\Delta k i m A$, showed the same SpeB activity as the wild type (Fig. 2, 3, and 7).

Three putative $\mathrm{K}^{+}$importers were identified in the $S$. pyogenes genome that could be involved in maintaining intracellular $\mathrm{K}^{+}$concentration. These importers are the 
KtrAB, Kup, and KimA systems. S. pyogenes Kup is an Escherichia coli Kup ortholog with $31.7 \%$ amino acid identity. E. coli Kup is a low-affinity $\mathrm{K}^{+}$importer and is believed to be the major $\mathrm{K}^{+}$importer system under acidic conditions $(28,29,33)$. In L. lactis, the Kup transporters are c-di-AMP binding proteins, and their potassium transport activities are inhibited upon binding to c-di-AMP (31). Gundlach et al. revealed that membrane protein $\mathrm{YdaO}$ in $B$. subtilis is a high-affinity $\mathrm{K}^{+}$importer, so they renamed the importer $\mathrm{KimA}\left(\mathrm{K}^{+}\right.$ importer A) (20). KimA in B. subtilis is inhibited by c-di-AMP at both the transcriptional and protein activity levels (30). A recent structural study revealed that $B$. subtilis KimA is a $\mathrm{K}^{+}$I $\mathrm{H}^{+}$symporter and also a member of the Kup family (34). S. pyogenes encodes a homolog of KimA, Spy_2088, with 20\% amino acid identity. In-frame deletion of ktrA, ktrB, kimA, or kup in the $\triangle$ dacA mutant revealed that only the deletion of $k t r B$ restored SpeB production equal to the wild-type level (Fig. 2). This suggests that the KtrAB system is the main $\mathrm{K}^{+}$importer maintaining cellular $\mathrm{K}^{+}$concentration under the in vitro growth conditions. Kup and KimA may have functions under different growth conditions, such as lower or higher osmotic or $\mathrm{pH}$ conditions than those tested in this study.

Even though $k t r B$ deletion in the $\triangle$ dacA mutant reverted many defective phenotypes of the $\triangle d a c A$ mutant, the ktrB deletion did not revert the loss of virulence of the $\triangle$ dacA mutant, indicating that other virulence traits regulated by c-di-AMP beyond SpeB production and resistance to stressors cannot be recovered by ktrB deletion alone. Thus, c-di-AMP in $S$. pyogenes appears to regulate multiple cellular pathways in addition to $\mathrm{K}^{+}$transport.

\section{MATERIALS AND METHODS}

Bacterial strains and media. S. pyogenes $\operatorname{HSC5}$ (emm genotype 14$)(35,36)$ was employed for all experiments, including strain construction. SF370 locus numbers (SPy_\#\#\#\#) are used as references for genes in HSC5 (37). Molecular cloning experiments utilized Escherichia coli DH5 $\alpha$ or TOP 10 (Invitrogen), which was cultured in Luria-Bertani broth. The routine culture of S. pyogenes employed Todd-Hewitt medium (BBL) supplemented with $0.2 \%$ yeast extract (Difco) (THY medium), and cells were grown at $37^{\circ} \mathrm{C}$ in sealed tubes without agitation. Unless otherwise indicated, $\mathrm{C}$ medium (38) was used to grow $S$. pyogenes for SpeB activity assay and RNA preparation for real-time qRT-PCR. Bacto agar (1.4\%, wt/vol; Difco) was added to make solid media. Cultures on solid media were incubated under the anaerobic condition created by a commercial product (GasPak; catalog no. 260678; $\mathrm{BBL})$. When appropriate, antibiotics were added to the media at the following concentrations if they are not specified: kanamycin, $50 \mu \mathrm{g} / \mathrm{ml}$ for E. coli and $500 \mu \mathrm{g} / \mathrm{ml}$ for S. pyogenes; erythromycin, $500 \mu \mathrm{g} / \mathrm{ml}$ for $E$. coli and $1 \mu \mathrm{g} / \mathrm{ml}$ for S. pyogenes.

Manipulation of DNA. Plasmid DNA was isolated via a commercial kit (Gene Elute plasmid miniprep kit; Sigma) and used to transform S. pyogenes or E. coli as described previously (39). Enzymes for DNA cloning and PCR were used according to the recommendations of the manufacturers. Chromosomal DNA was purified from S. pyogenes by using a standard kit (Wizard genomic DNA purification kit [Promega] or GenElute bacterial genomic DNA kit [Sigma]).

Strain construction. In-frame deletion mutations on chromosomal loci were generated by employing the shuttle vector with a temperature-sensitive replication origin, pJRS233 $(40,41)$. Briefly, PCR products immediately upstream and downstream of the deletion target gene were generated. The primers used to create the PCR products are listed in Table 1. These two PCR products were inserted into pJRS233 using the Gibson assembly (New England BioLabs) to create a deletion allele. A plasmid with a deletion allele, $\mathrm{p} \Delta k r t B, \mathrm{p} \Delta k \mathrm{kt} A, \mathrm{p} \Delta \mathrm{kim} A$, or $\mathrm{p} \Delta k u p$, was used to replace each target gene by the gene deletion method that employs the temperature-sensitive replication origin, as described previously (40, 41). The fidelity of all molecular constructs and gene deletions was confirmed by PCR and/or DNA sequencing (www.Psomagen.com).

Whole-genome sequencing. The whole-genome sequencing of the $\Delta d a c A \Delta k t r B^{\text {sup }}$ suppressor mutant was performed as described previously (18).

SpeB activity measurement using protease indicator plates. Strains were grown overnight in THY medium. The overnight cultures were serially diluted with fresh THY medium, and the diluted cells $(2 \mu \mathrm{l})$ were spotted onto protease indicator agar plates (C medium agar plates containing $2 \%$ skim milk). The protease indicator plates then were incubated anaerobically at $37^{\circ} \mathrm{C}$ for $24 \mathrm{~h}$ (or $48 \mathrm{~h}$ for extended incubation), and SpeB activity, which displays a clear zone around the spotted cells, was observed.

Measurement of biofilm formation degree. The degree of biofilm formation by strains was measured as described previously, with some modifications (42). Overnight cultures in $\mathrm{C}$ medium at $37^{\circ} \mathrm{C}$ were diluted at a ratio of 1:10 with fresh C medium, and $200 \mu \mathrm{l}$ of each diluted strain was added to the wells of flat-bottom 96-well polystyrene plates. Each plate was sealed with an optical tape and incubated for 48 to $72 \mathrm{~h}$ at room temperature. After incubation, the unbound cells were removed by rinsing the wells with distilled water four times, and then $200 \mu \mathrm{l}$ of crystal violet solution (3\%, wt/vol) was added to each well and incubated for $15 \mathrm{~min}$ at room temperature to stain the biofilm. The plates were rinsed four times with distilled water, and $200 \mu \mathrm{l}$ of ethanol (100\%) was added to each well to extract crystal 


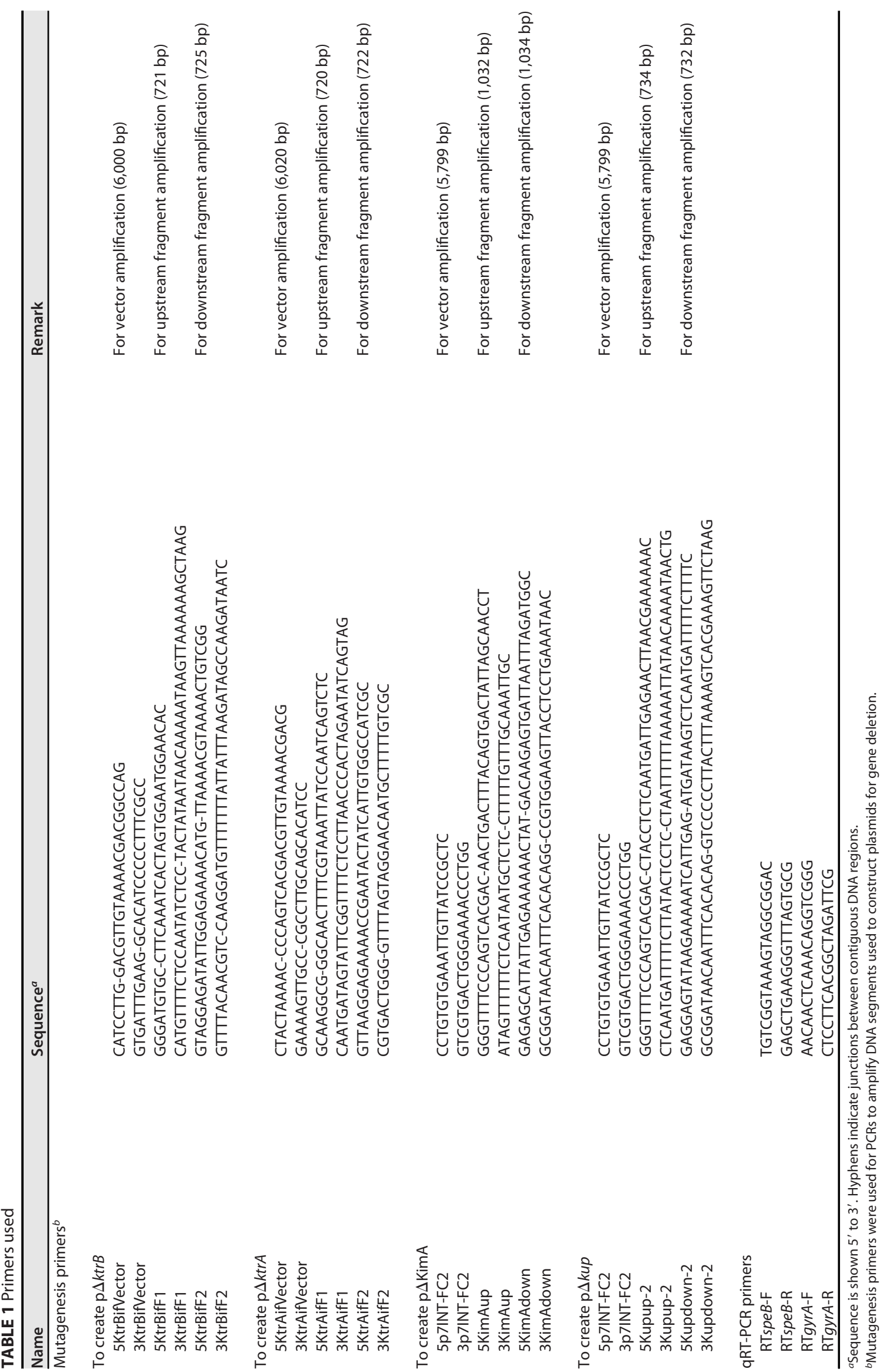


violet from cells. After 5 to $10 \mathrm{~min}$ of incubation, the solution $(100 \mu \mathrm{l})$ was transferred to new plates, and color intensity $\left(\mathrm{OD}_{600}\right)$ was measured with a 96-well plate reader (800 TS Absorbance Reader; BioTek). This experiment was performed in triplicate. The $\Omega e m m$ strain that does not express $M$ protein and is unable to form a biofilm (42) was used as a negative control.

Susceptibility to stress caused by salts, $\mathrm{pH}$, or oxygen radicals. The susceptibility of $S$. pyogenes to salt, $\mathrm{pH}$, and oxidative stress was assessed as described previously (18). These stress conditions were created by adding $\mathrm{KCl}$ or $\mathrm{NaCl}(0.1 \mathrm{M})$ into THY medium, adjusting the initial $\mathrm{pH}$ of THY or $\mathrm{C}$ medium to 6.0 with $2 \mathrm{~N} \mathrm{HCl}$, or adding methyl viologen into THY medium. For measuring the susceptibility to stress caused by salts or $\mathrm{pH}$, the optical density at $600 \mathrm{~nm}\left(\mathrm{OD}_{600}\right)$ of the cultures was measured at $8 \mathrm{~h}$ postinoculation, when $S$. pyogenes typically reaches the stationary phase. For measuring the susceptibility to oxidative stress, S. pyogenes cells were exposed to $50 \mathrm{mM}$ methyl viologen (MV) for $3 \mathrm{~h}$ in short-term exposure or $1.7 \mathrm{mM}$ for $18 \mathrm{~h}$ in long-term exposure. The survival rates were determined by comparing viable cell counts with and without MV treatment.

Susceptibility to PlyC. The susceptibility of $S$. pyogenes to the cell wall-hydrolyzing enzyme PlyC was examined as described previously (18). Briefly, S. pyogenes cells grown overnight were washed and resuspended in phosphate-buffered saline (PBS) $\left(\mathrm{OD}_{600}\right.$ of 1.0). PlyC $(3,000 \mathrm{U} / \mathrm{ml})$ and the cysteine protease inhibitor E-64 $(20 \mu \mathrm{M})$ were added into $1 \mathrm{ml}$ of the cell suspension. The cell suspension was incubated for $6 \mathrm{~h}$ at $37^{\circ} \mathrm{C}$, and then its turbidity $\left(\mathrm{OD}_{600}\right)$ was measured. This experiment was performed in duplicate.

Susceptibility to a sublethal concentration of ampicillin. The susceptibility of $S$. pyogenes to a sublethal concentration of the cell wall-targeting antibiotic ampicillin was examined as described previously (18). C medium containing ampicillin $(0.04 \mu \mathrm{g} / \mathrm{ml})$ was inoculated with a strain and incubated at $37^{\circ} \mathrm{C}$ overnight. The $\mathrm{OD}_{600}$ of the overnight culture $(\sim 18 \mathrm{~h}$ postinoculation) was measured to determine the final cell density. This experiment was performed in triplicate.

Murine subcutaneous infection assay. The ability of $S$. pyogenes strains to cause disease in soft tissues was evaluated using 6- to 8-week-old SKH1 hairless mice (Charles River Labs) as described previously (41). Briefly, each mouse was subcutaneously injected with approximately $1 \times 10^{7}$ CFU of S. pyogenes in $100 \mu \mathrm{l}$ into the right flank. The area of the lesion that formed was measured every $24 \mathrm{~h}$ by digital photography. Any differences in the areas of lesions between experimental groups were tested for significance by the Mann-Whitney $\mathrm{U}$ test. This study was carried out in strict accordance with the recommendations in the Guide for the Care and Use of Laboratory Animals of the National Institutes of Health (43). This animal study was approved by the Institutional Animal Care and Use Committee (IACUC) of Indiana State University (ISU).

The effect of ionophores on SpeB expression. S. pyogenes strains were grown overnight in THY medium. The cultures were then 10 -fold serially diluted with THY medium, and $2 \mu \mathrm{l}$ of each dilution was spotted on protease indicator plates containing $1 \mu \mathrm{g} / \mathrm{ml}$ valinomycin (V0627; Sigma) or $0.5 \mu \mathrm{g} / \mathrm{ml} \mathrm{grami-}$ cidin (G5002; Sigma) (stock solution: $10 \mathrm{mg} / \mathrm{ml}$ valinomycin in dimethyl sulfoxide and $10 \mathrm{mg} / \mathrm{ml}$ gramicidin in ethanol). The plates were incubated at $37^{\circ} \mathrm{C}$ anaerobically for 24 or $48 \mathrm{~h}$, and the clear zones by SpeB activity around the spotted cultures were observed.

Real-time qRT-PCR. Real-time qRT-PCR was conducted as described elsewhere (41). The primers for qRT-PCR are listed in Table 1. The gyrase A subunit gene $(g y r A)$ was used as the internal reference gene to normalize the expression level of a specific transcript between samples (44). The reported data represent the means and standard errors from three independent assays, each performed on a different day with newly extracted RNA.

Statistical testing. All statistical tests were performed using GraphPad Prism. Each statistical test applied to the experiments is described in the figure legends.

\section{ACKNOWLEDGMENTS}

We thank Razoanul Haque for his technical assistance in strain construction. We also thank Michael Caparon (Washington University) for sharing S. pyogenes strains.

\section{REFERENCES}

1. Hengge R, Grundling A, Jenal U, Ryan R, Yildiz F. 2016. Bacterial signal transduction by cyclic di-GMP and other nucleotide second messengers. J Bacteriol 198:15-26. https://doi.org/10.1128/JB.00331-15.

2. Kalia D, Merey G, Nakayama S, Zheng Y, Zhou J, Luo YL, Guo M, Roembke BT, Sintim HO. 2013. Nucleotide, c-di-GMP, c-di-AMP, cGMP, cAMP, (p) ppGpp signaling in bacteria and implications in pathogenesis. Chem Soc Rev 42:305-341. https://doi.org/10.1039/c2cs35206k.

3. Pesavento C, Hengge R. 2009. Bacterial nucleotide-based second messengers. Curr Opin Microbiol 12:170-176. https://doi.org/10.1016/j.mib.2009 .01 .007 .

4. Huynh TN, Choi PH, Sureka K, Ledvina HE, Campillo J, Tong L, Woodward JJ. 2016. Cyclic di-AMP targets the cystathionine beta-synthase domain of the osmolyte transporter OpuC. Mol Microbiol 102:233-243. https://doi .org/10.1111/mmi.13456.

5. Devaux L, Kaminski PA, Trieu-Cuot P, Firon A. 2018. Cyclic di-AMP in hostpathogen interactions. Curr Opin Microbiol 41:21-28. https://doi.org/10 .1016/j.mib.2017.11.007.
6. Zhang L, Li W, He ZG. 2013. DarR, a TetR-like transcriptional factor, is a cyclic di-AMP-responsive repressor in Mycobacterium smegmatis. J Biol Chem 288:3085-3096. https://doi.org/10.1074/jbc.M112.428110.

7. Bejerano-Sagie M, Oppenheimer-Shaanan Y, Berlatzky I, Rouvinski A, Meyerovich M, Ben-Yehuda S. 2006. A checkpoint protein that scans the chromosome for damage at the start of sporulation in Bacillus subtilis. Cell 125:679-690. https://doi.org/10.1016/j.cell.2006.03.039.

8. Mehne FM, Gunka K, Eilers H, Herzberg C, Kaever V, Stulke J. 2013. Cyclic di-AMP homeostasis in Bacillus subtilis: both lack and high level accumulation of the nucleotide are detrimental for cell growth. J Biol Chem 288:2004-2017. https://doi.org/10.1074/jbc.M112.395491.

9. Oppenheimer-Shaanan Y, Wexselblatt E, Katzhendler J, Yavin E, BenYehuda S. 2011. c-di-AMP reports DNA integrity during sporulation in Bacillus subtilis. EMBO Rep 12:594-601. https://doi.org/10.1038/embor.2011.77.

10. Corrigan RM, Abbott JC, Burhenne H, Kaever V, Grundling A. 2011. c-diAMP is a new second messenger in Staphylococcus aureus with a role in 
controlling cell size and envelope stress. PLoS Pathog 7:e1002217. https://doi.org/10.1371/journal.ppat.1002217.

11. Pozzi C, Waters EM, Rudkin JK, Schaeffer CR, Lohan AJ, Tong P, Loftus BJ, Pier GB, Fey PD, Massey RC, O'Gara JP. 2012. Methicillin resistance alters the biofilm phenotype and attenuates virulence in Staphylococcus aureus device-associated infections. PLoS Pathog 8:e1002626. https://doi.org/10 .1371 /journal.ppat.1002626.

12. Griffiths JM, O'Neill AJ. 2012. Loss of function of the GdpP protein leads to joint beta-lactam/glycopeptide tolerance in Staphylococcus aureus. Antimicrob Agents Chemother 56:579-581. https://doi.org/10.1128/AAC.05148-11.

13. Banerjee R, Gretes M, Harlem C, Basuino L, Chambers HF. 2010. A mecAnegative strain of methicillin-resistant Staphylococcus aureus with high-level beta-lactam resistance contains mutations in three genes. Antimicrob Agents Chemother 54:4900-4902. https://doi.org/10.1128/AAC.00594-10.

14. Zeden MS, Schuster CF, Bowman L, Zhong Q, Williams HD, Grundling A. 2018. Cyclic di-adenosine monophosphate (c-di-AMP) is required for osmotic regulation in Staphylococcus aureus but dispensable for viability in anaerobic conditions. J Biol Chem 293:3180-3200. https://doi.org/10 $.1074 / j b c . M 117.818716$.

15. Commichau FM, Stulke J. 2018. Coping with an essential poison: a genetic suppressor analysis corroborates a key function of c-di-AMP in controlling potassium ion homeostasis in Gram-positive bacteria. J Bacteriol 200:166-168. https://doi.org/10.1128/JB.00166-18.

16. Pham HT, Nhiep NTH, Vu TNM, Huynh TN, Zhu Y, Huynh ALD, Chakrabortti A, Marcellin E, Lo R, Howard CB, Bansal N, Woodward JJ, Liang ZX, Turner MS. 2018. Enhanced uptake of potassium or glycine betaine or export of cyclic-di-AMP restores osmoresistance in a high cyclic-di-AMP Lactococcus lactis mutant. PLoS Genet 14:e1007574. https://doi.org/10.1371/journal.pgen .1007574.

17. Woodward JJ, lavarone AT, Portnoy DA. 2010. c-di-AMP secreted by intracellular Listeria monocytogenes activates a host type I interferon response. Science 328:1703-1705. https://doi.org/10.1126/science.1189801.

18. Fahmi T, Faozia S, Port GC, Cho KH. 2019. The second messenger c-diAMP regulates diverse cellular pathways involved in stress response, biofilm formation, cell wall homeostasis, SpeB expression, and virulence in Streptococcus pyogenes. Infect Immun 87:147-149. https://doi.org/10 .1128/IAI.00147-19.

19. Gundlach J, Herzberg C, Hertel D, Thurmer A, Daniel R, Link H, Stulke J. 2017. Adaptation of Bacillus subtilis to life at extreme potassium limitation. mBio 8:e0861-17. https://doi.org/10.1128/mBio.00861-17.

20. Gundlach J, Herzberg C, Kaever V, Gunka K, Hoffmann T, Weiß M, Gibhardt J, Thürmer A, Hertel D, Daniel R, Bremer E, Commichau FM, Stülke J. 2017. Control of potassium homeostasis is an essential function of the second messenger cyclic di-AMP in Bacillus subtilis. Sci Signal 10: eaal3011. https://doi.org/10.1126/scisignal.aal3011.

21. Rocha R, Teixeira-Duarte CM, Jorge JMP, Morais-Cabral JH. 2019. Characterization of the molecular properties of KtrC, a second RCK domain that regulates a Ktr channel in Bacillus subtilis. J Struct Biol 205:34-43. https:// doi.org/10.1016/j.jsb.2019.02.002.

22. Vieira-Pires RS, Szollosi A, Morais-Cabral JH. 2013. The structure of the KtrAB potassium transporter. Nature 496:323-328. https://doi.org/10 $.1038 /$ nature 12055 .

23. Corrigan RM, Campeotto I, Jeganathan T, Roelofs KG, Lee VT, Grundling A. 2013. Systematic identification of conserved bacterial c-di-AMP receptor proteins. Proc Natl Acad Sci U S A 110:9084-9089. https://doi.org/10 $.1073 /$ pnas.1300595110.

24. Peng X, Zhang Y, Bai GC, Zhou XD, Wu H. 2016. Cyclic di-AMP mediates biofilm formation. Mol Microbiol 99:945-959. https://doi.org/10.1111/ mmi.13277.

25. Bai YL, Yang J, Zarrella TM, Zhang Y, Metzger DW, Bai GC. 2014. Cyclic diAMP impairs potassium uptake mediated by a cyclic di-AMP binding protein in Streptococcus pneumoniae. J Bacteriol 196:614-623. https://doi .org/10.1128/JB.01041-13.

26. Tesorero RA, Yu N, Wright JO, Svencionis JP, Cheng Q, Kim JH, Cho KH. 2013. Novel regulatory small RNAs in Streptococcus pyogenes. PLoS One 8: e64021. https://doi.org/10.1371/journal.pone.0064021.

27. David JM, Rajasekaran AK. 2015. Gramicidin A: a new mission for an old antibiotic. J Kidney Cancer VHL 2:15-24. https://doi.org/10.15586/jkcvhl .2015.21.

28. Bossemeyer D, Borchard A, Dosch DC, Helmer GC, Epstein W, Booth IR, Bakker EP. 1989. $\mathrm{K}^{+}$-transport protein TrkA of Escherichia coli is a peripheral membrane protein that requires other trk gene products for attachment to the cytoplasmic membrane. J Biol Chem 264:16403-16410. https://doi.org/10.1016/S0021-9258(19)84721-X.

29. Epstein W. 2003. The roles and regulation of potassium in bacteria. Prog Nucleic Acids Res Mol Biol 75:293-320. https://doi.org/10.1016/s0079 -6603(03)75008-9.

30. Gundlach J, Kruger L, Herzberg C, Turdiev A, Poehlein A, Tascon I, Weiss M, Hertel D, Daniel R, Hanelt I, Lee VT, Stulke J. 2019. Sustained sensing in potassium homeostasis: cyclic di-AMP controls potassium uptake by KimA at the levels of expression and activity. J Biol Chem 294:9605-9614. https://doi.org/10.1074/jbc.RA119.008774.

31. Quintana IM, Gibhardt J, Turdiev A, Hammer E, Commichau FM, Lee VT, Magni C, Stulke J. 2019. The KupA and KupB proteins of Lactococcus lactis IL1403 are novel c-di-AMP receptor proteins responsible for potassium uptake. J Bacteriol 201:e00028-19. https://doi.org/10.1128/JB .00028-19.

32. Szollosi A, Vieira-Pires RS, Teixeira-Duarte CM, Rocha R, Morais-Cabral JH. 2016. Dissecting the molecular mechanism of nucleotide-dependent activation of the KtrAB K $\mathrm{K}^{+}$transporter. PLoS Biol 14:e1002356. https://doi .org/10.1371/journal.pbio.1002356.

33. Trchounian A, Kobayashi H. 2000. $\mathrm{K}^{+}$uptake by fermenting Escherichia coli cells: $\mathrm{pH}$ dependent mode of the TrkA system operating. Biosci Rep 20:277-288. https://doi.org/10.1023/a:1026493024066.

34. Tascon I, Sousa JS, Corey RA, Mills DJ, Griwatz D, Aumuller N, Mikusevic V, Stansfeld PJ, Vonck J, Hanelt I. 2020. Structural basis of proton-coupled potassium transport in the KUP family. Nat Commun 11:626. https://doi .org/10.1038/s41467-020-14441-7.

35. Port GC, Paluscio E, Caparon MG. 2013. Complete genome sequence of emm type 14 Streptococcus pyogenes strain HSC5. Genome Announc 1: e00612-13. https://doi.org/10.1128/genomeA.00612-13.

36. Hanski E, Horwitz PA, Caparon MG. 1992. Expression of protein F, the fibronectin-binding protein of Streptococcus pyogenes JRS4, in heterologous streptococcal and enterococcal strains promotes their adherence to respiratory epithelial cells. Infect Immun 60:5119-5125. https://doi .org/10.1128/IAl.60.12.5119-5125.1992.

37. Ferretti JJ, McShan WM, Ajdic D, Savic DJ, Savic G, Lyon K, Primeaux C, Sezate S, Suvorov AN, Kenton S, Lai HS, Lin SP, Qian Y, Jia HG, Najar FZ, Ren Q, Zhu H, Song L, White J, Yuan X, Clifton SW, Roe BA, McLaughlin R. 2001. Complete genome sequence of an M1 strain of Streptococcus pyogenes. Proc Natl Acad Sci U S A 98:4658-4663. https://doi.org/10.1073/ pnas.071559398.

38. Lyon WR, Gibson CM, Caparon MG. 1998. A role for trigger factor and an rgg-like regulator in the transcription, secretion and processing of the cysteine proteinase of Streptococcus pyogenes. EMBO J 17:6263-6275. https://doi.org/10.1093/emboj/17.21.6263.

39. Caparon MG, Stephens DS, Olsen A, Scott JR. 1991. Role of M protein in adherence of group A streptococci. Infect Immun 59:1811-1817. https:// doi.org/10.1128/IAI.59.5.1811-1817.1991.

40. King KY, Horenstein JA, Caparon MG. 2000. Aerotolerance and peroxide resistance in peroxidase and PerR mutants of Streptococcus pyogenes. J Bacteriol 182:5290-5299. https://doi.org/10.1128/jb.182.19.5290-5299 2000.

41. Cho KH, Kang SO. 2013. Streptococcus pyogenes c-di-AMP phosphodiesterase, GdpP, influences SpeB processing and virulence. PLoS One 8: e69425. https://doi.org/10.1371/journal.pone.0069425.

42. Cho KH, Caparon MG. 2005. Patterns of virulence gene expression differ between biofilm and tissue communities of Streptococcus pyogenes. Mol Microbiol 57:1545-1556. https://doi.org/10.1111/j.1365-2958.2005.04786.x.

43. National Research Council. 2011. Guide for the care and use of laboratory animals, 8th ed. National Academies Press, Washington, DC.

44. Kang SO, Caparon MG, Cho KH. 2010. Virulence gene regulation by CvfA, a putative RNase: the CvfA-enolase complex in Streptococcus pyogenes links nutritional stress, growth-phase control, and virulence gene expression. Infect Immun 78:2754-2767. https://doi.org/10.1128/IAI.01370-09. 